\title{
A phenomenological cohesive model of ferroelectric fatigue
}

\author{
I. Arias ${ }^{b}$, S. Serebrinsky ${ }^{a, *}$, M. Ortiz ${ }^{\text {a }}$ \\ ${ }^{a}$ Division of Engineering and Applied Science, Graduate Aeronautical Laboratories, California Institute of Technology, \\ 1200 E. California Blvd., MS205-45, Pasadena, CA 91125, USA \\ b Laboratori de Càlcul Numèric, Departament de Matemàtica Aplicada III, Universitat Politècnica de Catalunya, Jordi Girona 1-3, Barcelona E-08034, Spain
}

Received 4 August 2005; received in revised form 5 October 2005; accepted 19 October 2005

Available online 5 January 2006

\begin{abstract}
We develop a phenomenological model of electro-mechanical ferroelectric fatigue based on a ferroelectric cohesive law that couples mechanical displacement and electric-potential discontinuity to mechanical tractions and surface-charge density. The ferroelectric cohesive law exhibits a monotonic envelope and loading-unloading hysteresis. The model is applicable whenever the changes in properties leading to fatigue are localized in one or more planar-like regions, modeled by the cohesive surfaces. We validate the model against experimental data for a simple test configuration consisting of an infinite slab acted upon by an oscillatory voltage differential across the slab and otherwise stress free. The model captures salient features of the experimental record including: the existence of a threshold nominal field for the onset of fatigue; the dependence of the threshold on the applied-field frequency; the dependence of fatigue life on the amplitude of the nominal field; and the dependence of the coercive field on the size of the component, or size effect. Our results, although not conclusive, indicate that planar-like regions affected by cycling may lead to the observed fatigue in tetragonal PZT.
\end{abstract}

(c) 2005 Acta Materialia Inc. Published by Elsevier Ltd. All rights reserved.

Keywords: Ferroelectricity; Fatigue; Fracture; Nucleation; Cohesive

\section{Introduction}

Ferroelectric materials are extensively used in a variety of sensor and actuator applications, where the coupling between mechanical and electrical fields is of primary interest. They are also a promising set of materials for improved dynamic as well as non-volatile memory devices, where only the electrical properties are directly exploited. However, ferroelectrics are brittle, and their low fracture toughness (in the order of $1 \mathrm{MPa} \mathrm{m}{ }^{1 / 2}$ ) makes them susceptible to cracking. In addition, ferroelectric materials exhibit electrical fatigue (loss of switchable polarization) under cyclic electrical loading and, due to the strong electro-mechanical coupling, sometimes mechanical fatigue as

\footnotetext{
* Corresponding author. Tel.: +1 626395 3282; fax: +1 6264492677.

E-mail address: serebrin@caltech.edu (S. Serebrinsky).
}

well. Conversely, the propagation of fatigue cracks hinders the performance of the devices and raises serious reliability concerns.

Ferroelectric fatigue is caused by a combination of electrical, mechanical and electro-chemical processes, each of which has been claimed to be responsible for fatigue [1]. Several electro-chemical mechanisms have been posited as the likely cause of polarization fatigue [2], but no general consensus appears to have emerged as yet. Fatigue mechanisms variously include processes of distributed damage over the bulk (see, e.g., Refs. $[3,4])$ and processes of localized damage, including microcracks and ferroelectric-electrode interfaces (see, e.g., Refs. [5-7]). The proposed mechanisms include domainwall pinning and inhibition of reversed domain nucleation $[8,9,2]$. The former mechanism is thought to play a dominant role in the bulk, whereas the latter mechanism is thought to operate primarily at or near electrode 
interfaces [10]. The relative roles of these and other mechanisms may depend on the frequency of the applied field [10]. At the atomic level, oxygen vacancies are likely to promote fatigue, e.g., by migrating under the action of the electric field to form extended defects that pin domain walls [11-13]. Purely mechanical mechanisms, such as microcracking, are also likely to play some role. Thus, fatigued ceramic specimens often contain scattered microcracks of size comparable to that of the grains $[14,15]$. Profuse microcrack clouds have been observed at the specimen edges and surrounding macroscopic cracks [16]. Macroscopic crack patterns are present in some fatigued ceramic specimens [17]. Severe cracking was also observed in barium titanate single crystals subjected to cyclic bipolar electric load [18]. Modeling work suggests that microcracking is indeed a cause of loss of polarization [6]

Experiments on crack propagation have been reported for samples loaded electrically, mechanically, or under combined loading, cyclic or static [19-22]. It is not uncommon for different experiments to lead to apparently contradictory conclusions [23], a testament to the complexity of the phenomenon of ferroelectric fatigue.

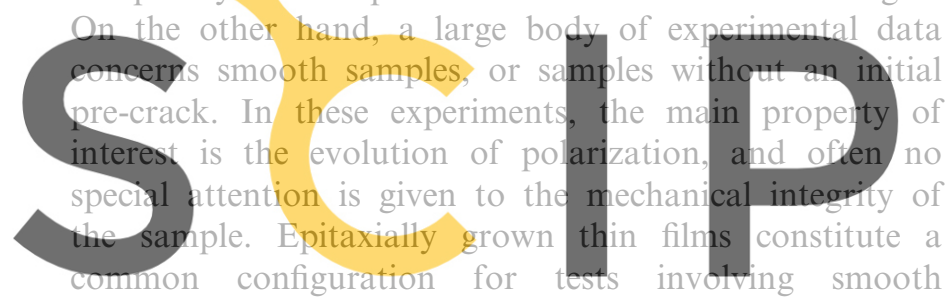
samples [24]

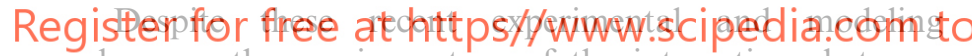
advances, the precise nature of the interactions between fracture, deformation and defect structures underlying ferroelectric fatigue is in need of further elucidation, and a physics-based multiscale model enabling the prediction of the fatigue life of ferroelectric devices is yet to emerge. Therefore, there remains a need for phenomenological and empirical models that can be experimentally validated and used in engineering design. In this vein, cohesive theories provide an effective means of modeling fatigue-crack nucleation and growth for arbitrary crack and specimen geometries and loading histories [25,26]. As noted by Nguyen et al. [25], the essential feature that a cohesive law must possess in order to model fatigue crack growth is loading-unloading hysteresis. By this simple but essential device, cohesive models of mechanical fatigue have been shown to account for deviations from Paris's law in metals such as are observed for short cracks and overloads [25]; and to predict fatigue-crack nucleation in smooth-surface metallic specimens [26]. The appealing feature of cohesive models of fatigue is that a single mechanistic, albeit empirical, model applies to nucleation and propagation, short and long cracks, and arbitrary loading histories.

Conventional cohesive models of fracture seek to describe the relation between cohesive tractions and open- ing displacements at the tip of a crack. Working by analogy, Gao et al. [27-29] have proposed the use of Dugdale-like models for the electric displacement in ferroelectric materials. In this paper, we further extend the concept of cohesive surface to ferroelectric materials by understanding it to be a surface of both mechanical opening displacement and electric-potential discontinuity. Correspondingly, we extend the concept of cohesive law to mean a general relation between the work-conjugate pairs: mechanical displacement and electric-potential discontinuity; and mechanical tractions and surface-charge density. Following Nguyen et al. [25], in order to model ferroelectric fatigue we endow the ferroelectric cohesive law with loading-unloading hysteresis. In order to simplify modeling, following [30] we make the additional assumption that the cohesive law derives from a potential that depends on a scalar effective electro-mechanical jump, which is a weighted norm of mechanical opening displacements and electric-potential jump.

Using a simple Smith-Ferrante monotonic envelope and an exponential-decay law of loading-unloading hysteresis, we show that the model is able to qualitatively capture salient features of the experimental record includ-

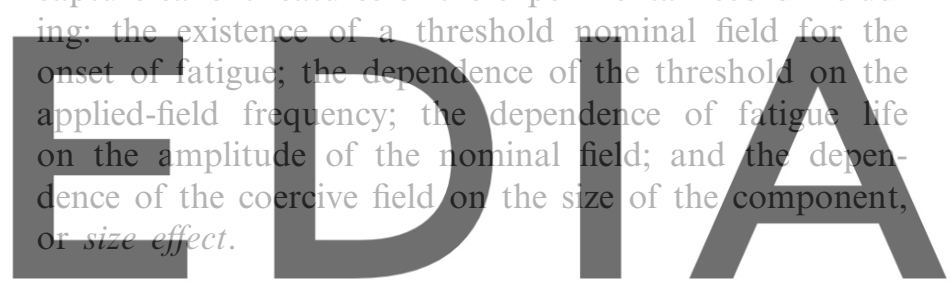

2. Electro-mechanical cohesive laws

\section{download the version without the watermark}

The essential structure of cohesive laws in ferroelectrics may be elucidated by recourse to a conventional Coleman-

Noll argument (cf., [30] for an application to mechanical decohesion). In particular, the pairing between stress-like and deformation-like variables is determined by the internal-power identity or, equivalently, by the virtual-work identity. For a dielectric solid, this identity is [31-33]

$$
\begin{aligned}
& \int_{\Omega}(\boldsymbol{\sigma}: \delta \boldsymbol{\epsilon}-\boldsymbol{D} \cdot \delta \boldsymbol{E}) \mathrm{d} V \\
& \quad=\int_{\Omega}(\boldsymbol{b} \cdot \delta \boldsymbol{u}-\rho \delta \phi) \mathrm{d} V+\int_{\partial \Omega}\left(\boldsymbol{t} \cdot \delta \boldsymbol{u}-\omega_{\mathrm{f}} \delta \phi\right) \mathrm{d} S,
\end{aligned}
$$

where $\Omega$ is the spatial domain occupied by the solid and $\partial \Omega$ is its boundary; $\boldsymbol{\sigma}$ is the stress;

$\boldsymbol{\epsilon}=\frac{1}{2}\left(\nabla \boldsymbol{u}+\nabla \boldsymbol{u}^{\mathrm{T}}\right)$

is the strain; $\boldsymbol{D}$ is the electric displacement;

$\boldsymbol{E}=-\nabla \phi$

is the electric field; $\boldsymbol{b}$ is the body force; $\boldsymbol{u}$ is the displacement; $\rho$ is the free charge density; $\phi$ is the electric potential; $\boldsymbol{t}=\boldsymbol{\sigma} \cdot \boldsymbol{n}$ is the surface traction; and $\omega_{\mathrm{f}}=-\boldsymbol{D} \cdot \boldsymbol{n}$ is the free surface charge per unit area. For a solid with a surface of discontinuity $\Gamma$, or cohesive surface, we have 


$$
\begin{aligned}
\delta W_{\mathrm{ext}}= & \int_{\Omega}(\boldsymbol{b} \cdot \delta \boldsymbol{u}-\rho \delta \phi) \mathrm{d} V+\int_{\partial \Omega}\left(\boldsymbol{t} \cdot \delta \boldsymbol{u}-\omega_{\mathrm{f}} \delta \phi\right) \mathrm{d} S \\
= & \int_{\Omega}(\boldsymbol{b} \cdot \delta \boldsymbol{u}-\rho \delta \phi) \mathrm{d} V+\int_{\partial \Omega}[(\boldsymbol{n} \cdot \boldsymbol{\sigma}) \cdot \delta \boldsymbol{u}+(\boldsymbol{n} \cdot \boldsymbol{D}) \delta \phi] \mathrm{d} S \\
= & \int_{\Omega}(\boldsymbol{b} \cdot \delta \boldsymbol{u}-\rho \delta \phi) \mathrm{d} V+\int_{\Omega^{ \pm}}[\nabla \cdot(\boldsymbol{\sigma} \cdot \delta \boldsymbol{u}) \\
& +\nabla \cdot(\boldsymbol{D} \delta \phi)] \mathrm{d} V+\int_{\Gamma}[(\boldsymbol{n} \cdot \boldsymbol{\sigma}) \cdot \llbracket \delta \boldsymbol{u} \rrbracket+(\boldsymbol{n} \cdot \boldsymbol{D}) \llbracket \delta \phi \rrbracket] \mathrm{d} S
\end{aligned}
$$

and consequently

$$
\begin{aligned}
\delta W_{\text {int }}= & \int_{\Omega^{ \pm}}(\boldsymbol{\sigma}: \delta \boldsymbol{\epsilon}-\boldsymbol{D} \cdot \delta \mathbf{E}) \mathrm{d} V+\int_{\Gamma}[(\boldsymbol{n} \cdot \boldsymbol{\sigma}) \cdot \delta \llbracket \boldsymbol{u} \rrbracket \\
& +(\boldsymbol{n} \cdot \boldsymbol{D}) \delta \llbracket \phi \rrbracket] \mathrm{d} S .
\end{aligned}
$$

For monotonic loading of the cohesive surface, these workconjugacy relations naturally suggest a free energy per unit surface, or cohesive potential, of the form $\Phi(\llbracket u \rrbracket, \llbracket \phi \rrbracket, q)$ such that

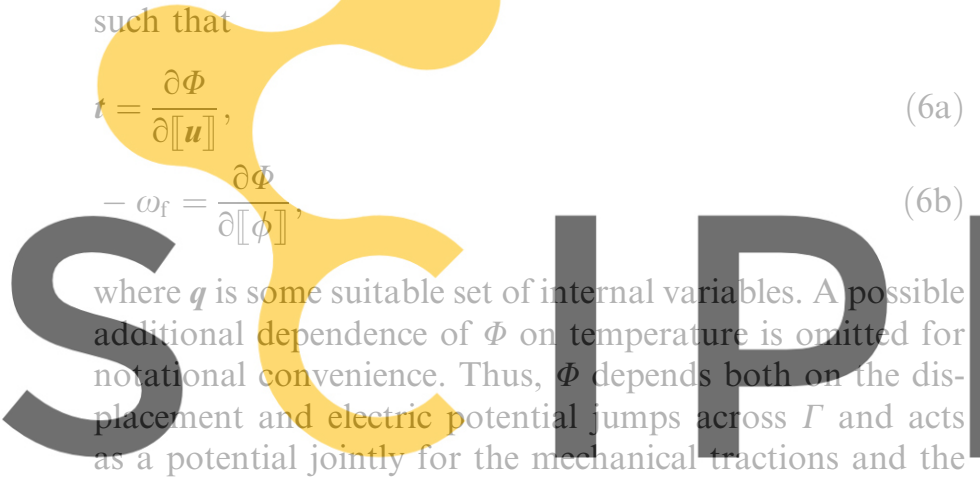
surface-charge density

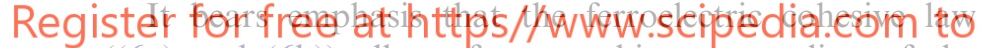
((6a) and (6b)) allows for an arbitrary coupling of the mechanical and electrical fields. It should also be carefully noted that the ferroelectric cohesive law describes the physics of mechanical or electrical decohesion and does not presume a particular form of the constitutive law governing the behavior in the bulk. In particular, it is possible to apply the ferroelectric cohesive law ((6a) and (6b)) in conjunction with Landau-Ginzburg-Devonshire models of domain switching (cf., e.g., Ref. [34]). The detailed boundary conditions on the crack faces are thought to greatly affect the fracture behavior of electrically driven crack growth [35]. For instance, partial discharge or charge separation effects have been suggested to play an important role in the vicinity of the crack tip [36]. The ferroelectric cohesive law ((6a) and (6b)) provides a useful framework for modeling those phenomena as well. Moreover, the ferroelectric cohesive law encodes the physics of decohesion, and thus can be tailored to represent any of the localized mechanisms of ferroelectric fatigue.

\subsection{Ferroelectric fatigue cohesive law}

As noted by Nguyen et al. [25], reversible cohesive laws do not predict crack advance under cyclic loading and, therefore, are insufficient for modeling fatigue. Instead, for a cohesive law to predict fatigue it must be irreversible and account for loading-unloading hysteresis. Loadingunloading irreversibility may be built into a cohesive law by means of the internal variable formalism (cf., e.g., Ref. [30]). The loading-unloading hysteresis model developed subsequently extends that of Nguyen et al. [25] to ferroelectric fatigue.

The modeling process is greatly simplified by the assumption that the cohesive potential $\Phi$ depends on the displacement and electric-potential jumps only through the effective electro-mechanical jump $[37,30]$

$\delta=\sqrt{\delta_{n}^{2}+\beta^{2} \delta_{s}^{2}+\gamma^{2} \psi^{2}}$,

i.e., by assuming

$\Phi=\Phi(\delta, q)$,

where we write

$\delta=\llbracket \boldsymbol{u} \rrbracket$,

$\delta_{n}=\delta \cdot \boldsymbol{n}$,

$\boldsymbol{\delta}_{n}=\delta_{n} \boldsymbol{n}$

$\delta=\sqrt{\delta^{2}-\delta^{2}}$

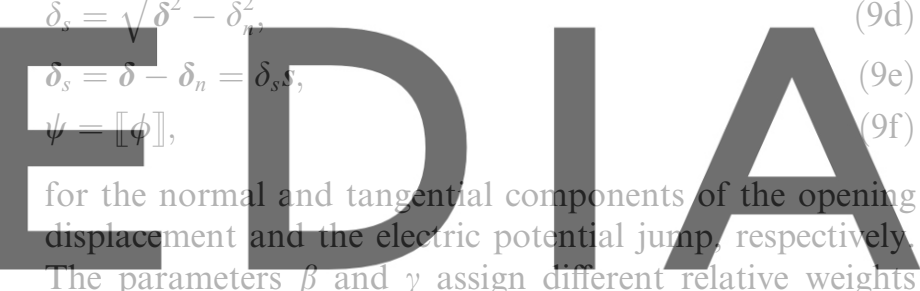
to normal and tangential opening displacements, thus dif-

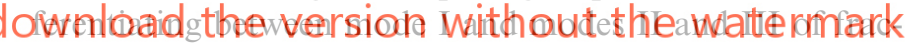
ture; and to opening displacements and the electric potential jump, thus differentiating between mechanical and electrical fatigue.

An effective electro-mechanical flux may also be defined as

$\sigma=\frac{\partial \Phi}{\partial \delta}$

Using the chain rule, (6a) and (6b) evaluate to

$\boldsymbol{\tau}=\frac{\sigma}{\delta}\left[\left(1-\beta^{2}\right) \delta_{n} \boldsymbol{n}+\beta^{2} \boldsymbol{\delta}\right]=\frac{\sigma}{\delta}\left(\delta_{n} \boldsymbol{n}+\beta^{2} \delta_{s} \boldsymbol{s}\right)=\tau_{n} \boldsymbol{n}+\tau_{s} \boldsymbol{s}$,

$-\omega_{\mathrm{f}}=\frac{\sigma}{\delta} \gamma^{2} \psi$.

We also note that

$\sigma=\sqrt{\tau_{n}^{2}+\tau_{s}^{2} / \beta^{2}+\omega_{\mathrm{f}}^{2} / \gamma^{2}}$.

Next we further specialize the preceding framework along the lines of the cohesive model of fatigue crack nucleation and growth proposed by Serebrinsky and Ortiz [26] and by Nguyen et al. [25]. The essential feature to include in the model is loading-unloading hysteresis. The specific scheme chosen to build hysteresis into the model is illustrated in Fig. 1. Monotonic loading is characterized by a cohesive 


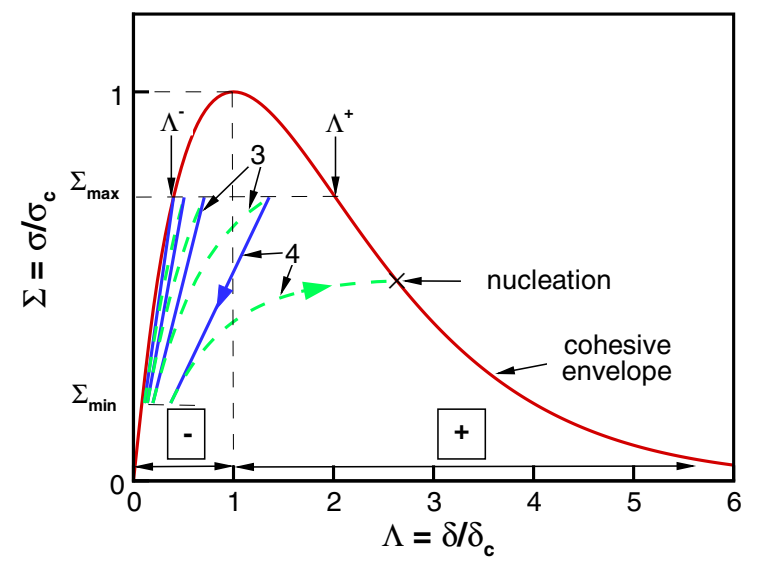

Fig. 1. Cyclic behavior predicted by the model and conventional definition of fatigue initiation.

law $\sigma(\delta)$, referred to as the monotonic envelope, which is characterized by the critical electro-mechanical flux $\sigma_{\mathrm{c}}$ and the critical electro-mechanical jump $\delta_{\mathrm{c}}$, and possibly an initial threshold $\sigma_{\text {th. }}$. In contrast, cyclic loading is hysteretic and governed by the loading-unloading law:

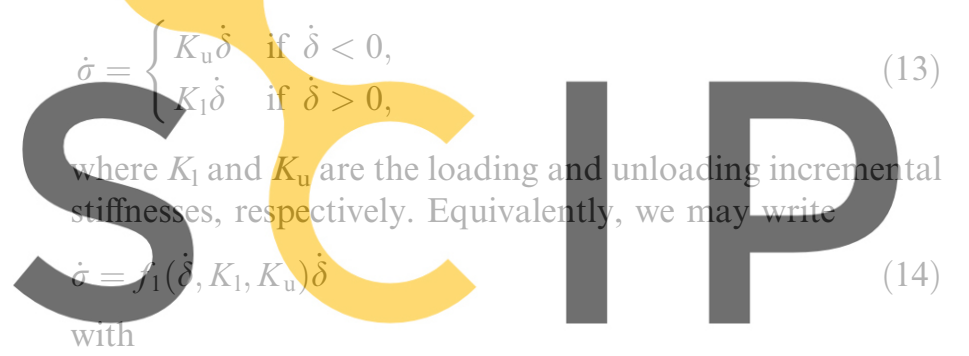

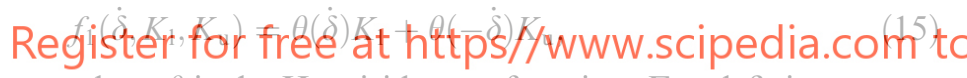
where $\theta$ is the Heaviside step function. For definiteness, we assume unloading to take place towards the origin and $K_{\mathrm{u}}$ to be constant during unloading, Fig. 1. The value of $K_{\mathrm{u}}$ during an unloading event is determined by the values of $\sigma$ and $\delta$ at the unloading point. Upon reloading, we additionally suppose that the reloading slope $K_{1}=\mathrm{d} \sigma / \mathrm{d} \delta$ decreases with increasing electro-mechanical jump as a result of interfacial degradation mechanisms occurring at the microscale. During unloading, a partial recovery of $K_{1}$ is also allowed for. For definiteness, we take

$\dot{K}_{1}= \begin{cases}\left(K_{1}-K_{\mathrm{u}}\right) \dot{\delta} / \delta_{\mathrm{a}} & \text { if } \dot{\delta}<0, \\ -K_{1} \dot{\delta} / \delta_{\mathrm{a}} & \text { if } \dot{\delta}>0,\end{cases}$

where $\delta_{\mathrm{a}}$ is an intrinsic length of the material. Equivalently, we may write

$\dot{K}_{1}=f_{2}\left(\dot{\delta}, K_{1}, K_{\mathrm{u}}\right) \dot{\delta} / \delta_{\mathrm{a}}$

with

$f_{2}\left(\dot{\delta}, K_{1}, K_{\mathrm{u}}\right)=-\theta(\dot{\delta}) K_{1}+\theta(-\dot{\delta})\left(K_{1}-K_{\mathrm{u}}\right)$.

Thus, $\boldsymbol{q}=\left(K_{\mathrm{u}}, K_{1}\right)$ may be regarded as the internal variables of the model. An appealing feature of the model is the small number of parameters, namely: the constants defining the monotonic envelope, such as tensile strength and toughness; the coupling constants $\beta$ and $\gamma$; and the intrinsic length $\delta_{\mathrm{a}}$.

The cyclic behavior predicted by the model just outlined is shown schematically in Fig. 1 for cycling between constant maximum and minimum effective electro-mechanical fluxes. Thus, for monotonic loading the cohesive surface follows the monotonic envelope. Upon cyclic loading, the cohesive stiffness of the surface degrades steadily and, after a certain number of cycles, the $(\sigma(t), \delta(t))$ curve meets the monotonic envelope. We identify this event with the end of the fatigue life of the material. Indeed, once the curve $(\sigma(t), \delta(t))$ meets the monotonic envelope, the material can no longer sustain a loading cycle of the same amplitude and, consequently, fails catastrophically under load control.

\subsection{Bulk behavior}

A closed set of governing equations may be obtained by appending a suitable bulk energy density to the cohesive model just described and considering the corresponding gradient flow. Following Zhang and Bhattacharya [38], we assume a bulk energy density of the form

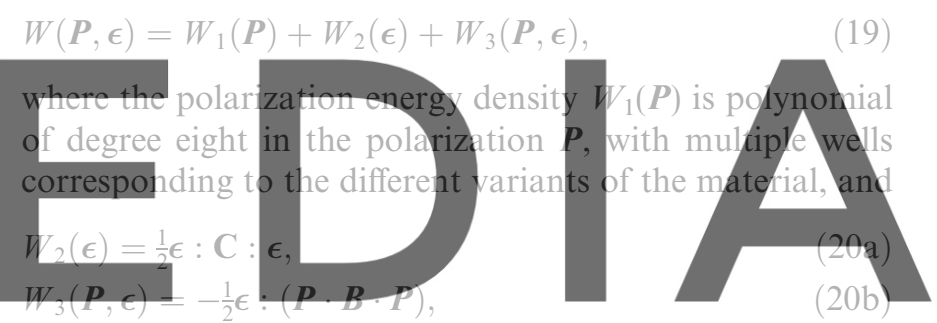

are the strain and mixed energy densities, respectively. Foldown lad the versign wathout the watermark the total energy of the body to be of the form

$E[\boldsymbol{P}, \boldsymbol{u}, \phi]=\int_{\Omega}\left(W(\boldsymbol{P}, \boldsymbol{\epsilon})+\frac{\epsilon_{0}}{2}|\nabla \phi|^{2}\right) \mathrm{d} \Omega$,

where $W$ is the bulk energy density and $\epsilon_{0}=8.854 \times$ $10^{-12} \mathrm{~F} / \mathrm{m}$ is the permeability of vacuum and the energy of domain walls is neglected for simplicity. A gradient flow of this energy results in the time-dependent GinzburgLandau equations

$\mu \dot{\boldsymbol{P}}=-\frac{\partial W}{\partial \boldsymbol{P}}(\boldsymbol{P}, \boldsymbol{\epsilon})+\boldsymbol{E}$,

$\nabla \cdot\left(\boldsymbol{P}+\epsilon_{0} \boldsymbol{E}\right)=0$,

$\nabla \cdot \frac{\partial W}{\partial \boldsymbol{\epsilon}}(\boldsymbol{P}, \boldsymbol{\epsilon})=\mathbf{0}$,

where $1 / \mu$ is the mobility. In these equations, we recognize an equation of evolution for the polarization $\boldsymbol{P}$, Gauss's law, and the equation of mechanical equilibrium, respectively. At equilibrium $\dot{\boldsymbol{P}}=0$ and Eq. (22) reduces to

$-\frac{\partial W}{\partial \boldsymbol{P}}(\boldsymbol{P}, \boldsymbol{\epsilon})+\boldsymbol{E}=0$,

which is also obtained in the formal limit of $\mu \rightarrow 0$. Eqs. (22)-(24), in conjunction with (3) and (2), define a closed 
initial-boundary-value problem for the polarization $\boldsymbol{P}$, the electro-static potential $\phi$ and the mechanical displacement $\boldsymbol{u}$.

\section{Experimental validation}

In order to make contact with experiment and assess the validity of the model, we consider a simple test configuration consisting of an infinite slab of thickness $u$ acted upon by an oscillatory voltage differential $\Delta \phi_{T}$ across the slab and otherwise stress free. The mid plane of the slab is a weak interface governed by the cohesive model proposed in Section 2. In order to simplify the analysis, we assume all fields to be uniform outside the cohesive interface. This assumption has the effect of reducing the initial-boundaryvalue problem (22)-(24) to a simple set of ordinary differential equations in time. Taking the cohesive plane to coincide with the $\left(x_{1}, x_{2}\right)$-coordinate plane and assuming uniaxial strain conditions, $\epsilon_{11}=\epsilon_{22}=\epsilon_{12}=\epsilon_{13}=\epsilon_{23}=0$, the various bulk energy densities reduce to

$W_{1}\left(P_{3}\right)=\frac{a_{1}}{2} P_{3}^{2}+\frac{a_{2}}{4} P_{3}^{4}+\frac{a_{3}}{6} P_{3}^{6}$, (26a)

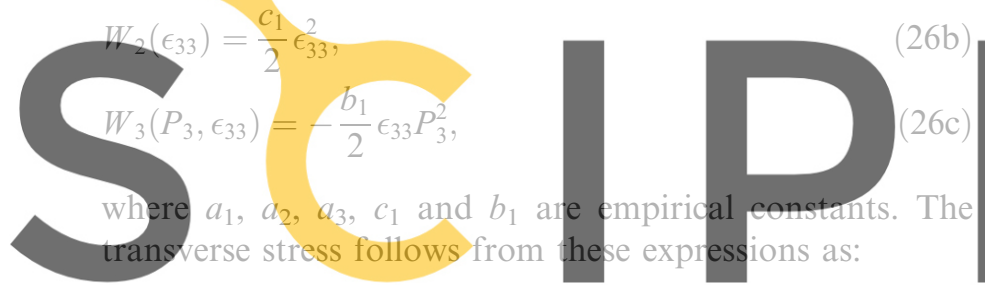

\section{Register for free àt https//www.scipedia.com to}

The stress-free condition $\sigma_{33}=0$ then gives the transverse strain as

$\epsilon_{33}=\frac{b_{1}}{2 c_{1}} P_{3}^{2}$.

Using this identity, (22) reduces to

$\mu \dot{P}_{3}+a_{1} P_{3}+\left(a_{2}-\frac{b_{1}^{2}}{2 c_{1}}\right) P_{3}^{3}+a_{3} P_{3}^{5}=E_{3}$.

From Eq. (23) and the boundary conditions, at the cohesive interface, we additionally have

$-\omega_{\mathrm{f}}=P_{3}+\epsilon_{0} E_{3}$,

$\psi=E_{3} u+\Delta \phi_{T}$.

Finally, the coupled electro-mechanical cohesive law specializes to

$\dot{\sigma}=f_{1}\left(\dot{\delta}, K_{1}, \sigma / \delta\right) \dot{\delta}$,

$\dot{K}_{1}=f_{2}\left(\dot{\delta}, K_{1}, \sigma / \delta\right) \dot{\delta}$

$\gamma^{2}\left(\frac{P_{3} u-\epsilon_{0} \Delta \phi_{T}}{\sigma u \gamma^{2}-\epsilon_{0} \delta}\right)^{2}=1$ where

$\delta=\gamma|\psi|$,

$\sigma=\frac{1}{\gamma}|\omega|$.

In calculations, we enforce the constraint (31c) in differential form and solve the resulting system of ordinary differential equations in time by means of a fourth-order Runge-Kutta algorithm. In implementing this algorithm, care must be exercised in order to resolve ambiguities in the selection of branches and the handling of singularities in the response functions as $\delta$ tends to zero. In the sequel, $P=P_{3}$ and similarly for other vector quantities, and $\epsilon=\epsilon_{33}$.

For sufficiently high applied field, the calculated response exhibits characteristic hysteresis loops in a $P-E$ plot, and butterfly loops in a $\epsilon-E$ plot. These loops are shown in Fig. 2 for several values of the peak electric field $E_{\mathrm{p}}$ and the applied-field frequency $f$. Several aspects of the bulk response are noteworthy. Thus, below a minimum value of $E_{\mathrm{p}}$ no switching occurs, and the sign of the polarization

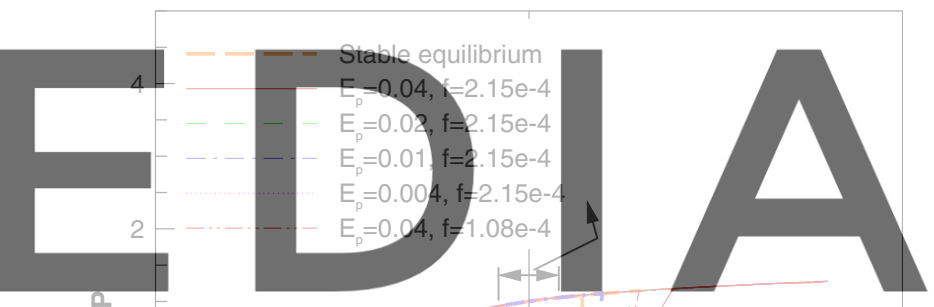

ㄴ.
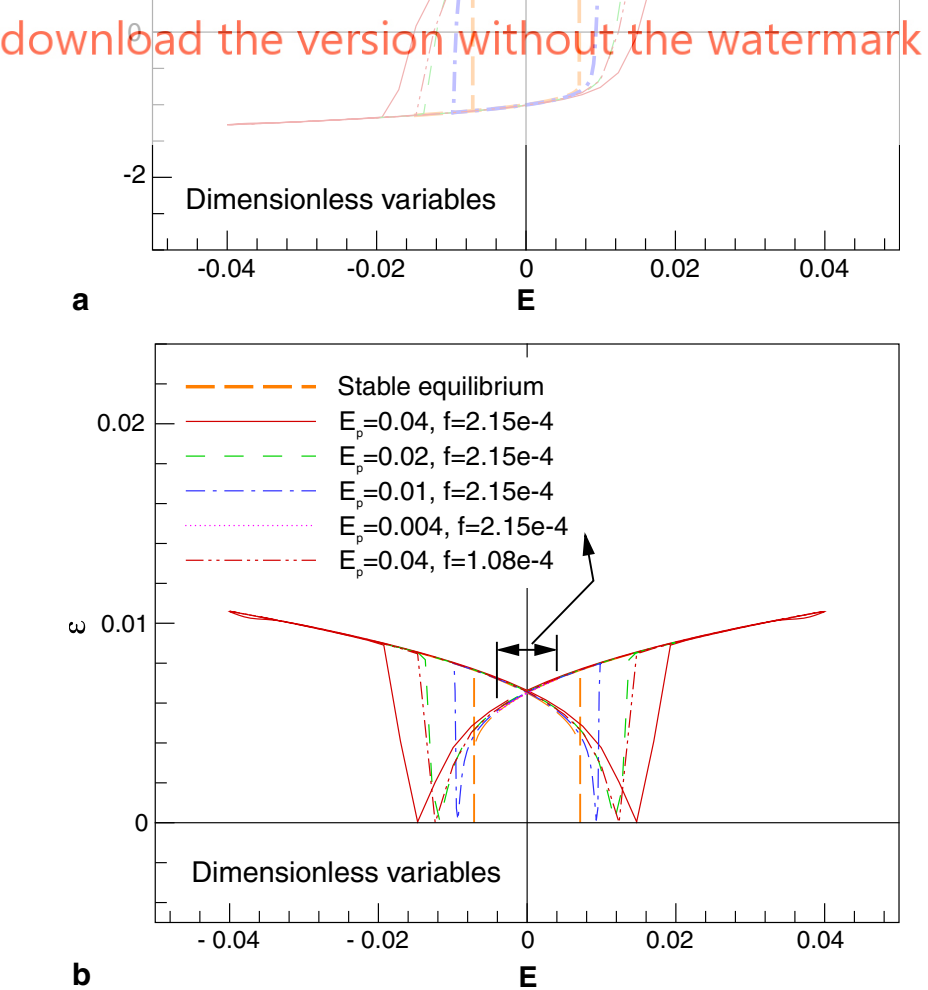

Fig. 2. Bulk response for different field amplitudes and frequencies. (a) $P$ $E$ hysteresis loops. (b) $\epsilon-E$ butterfly loops. 
remains constant in time. The rate-dependency introduced by the time-dependent Ginzburg-Landau equation is evident in Fig. 2. In the limit of $\mu=0$ or, equivalently, of a small frequency of the applied field, this rate dependency is removed and an equilibrium loop is obtained. For nonzero $\mu$, the bulk response depends on the applied-field frequency.

The predictions of the model in the example just described can now be compared with experimental data by way of validation. In order to facilitate comparisons for different materials, we introduce the following normalization constants for the variables: polarization, $P_{\text {ref }}$; stress, elastic constants, $c_{\text {ref }}$; permittivity, $\epsilon_{0 \text { ref }}=P_{\text {ref }}^{2} / c_{\text {ref }}$; electric field, $E_{\text {ref }}=P_{\text {ref }} / \epsilon_{0 \text { ref }}$; thickness, displacements, $L_{\text {ref }}=\delta_{\text {a }}$; potential, $\phi_{\text {ref }}=E_{\text {ref }} L_{\text {ref }}$; time $t_{\text {ref }}=\mu P_{\text {ref }}^{2} / c_{\text {ref }}$; energy density coefficients, $b_{\text {ref }}=a_{1 \text { ref }}=c_{\text {ref }} / P_{\text {ref }}, \quad a_{2 \text { ref }}=a_{1 \text { ref }} / P_{\text {ref }}^{2}$, $a_{3 \text { ref }}=a_{1 \text { ref }} / P_{\text {ref }}^{4} ;$ coefficient $\gamma_{\text {ref }}=P_{\text {ref }} / c_{\text {ref }} ;$ cohesive stiffness $K_{\text {ref }}=c_{\text {ref }} / L_{\text {ref }}$. Dimensionless variables are used in the calculations, and the parameters and material constants are summarized in Table 1. We have selected material constants for the bulk model readily available from the literature [38], which correspond to $\mathrm{BaTiO}_{3}$. The initial conditions are $P_{30}=1, K_{10}=2311$, and $\delta_{0}$ and $\sigma_{0}$ are cho-

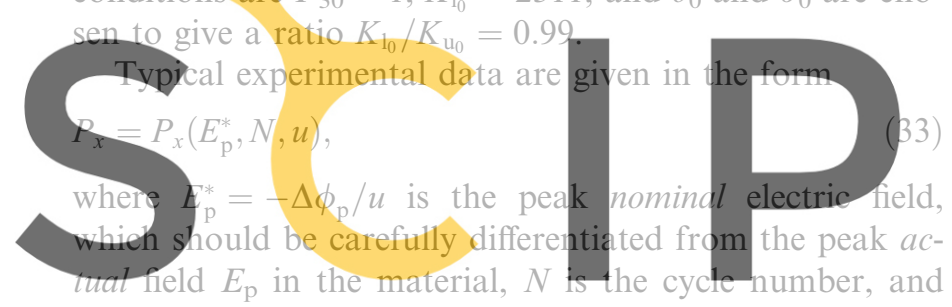

$P_{x}$ is a measure of the polarization state of the material,

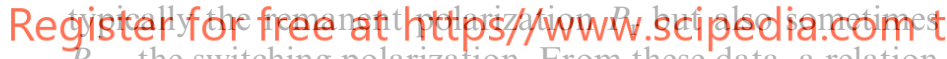

$P_{\text {sw }}$, the switching polarization. From these data, a relation $E_{\mathrm{f}}^{*}(N, u)$, or the inverse relation $N_{\mathrm{f}}\left(E_{\mathrm{p}}^{*}, u\right)$, can be derived by solving the equation

$\frac{P_{x}\left(E_{\mathrm{p}}^{*}, N, u\right)}{P_{x}\left(E_{\mathrm{p}}^{*}, 1, u\right)}=1-C$,

where $C$ is the loss of polarization due to fatigue. We shall take these fatigue maps as the basis for the validation of the model.

As expected, the predicted fatigue behavior depends critically on whether switching occurs. In the presence of switching, the interface degrades upon cycling and the peak value of $\delta$ increases monotonically with the number of cycles, Fig. 3. The increase in $\delta$ is initially slow and accelerates markedly in the last stages of the fatigue life of the material. The rate of damage accumulation increases with the amplitude of the nominal field. Correspondingly, as the interface degrades the switching capability and the actuation strain are impaired.

Table 1

Values of the model parameters

\begin{tabular}{llllllll}
\hline$a_{1}$ & $a_{2}$ & $a_{3}$ & $b_{1}$ & $c_{1}$ & $\epsilon_{0}$ & $\gamma$ & $\mu$ \\
\hline-0.007 & -0.009 & 0.0261 & 1.4282 & 185 & 0.131 & 800 & 1 \\
\hline
\end{tabular}

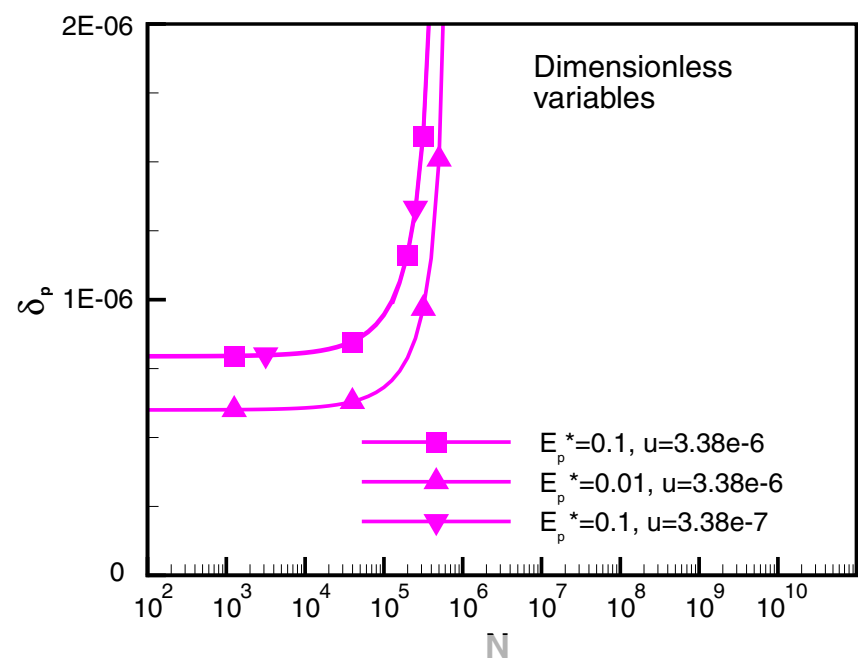

Fig. 3. Evolution with number of cycles of the peak effective opening displacement $\delta_{\mathrm{p}}$.

The calculated fatigue map, for small frequencies for which rate-dependency is negligible, is shown in Fig. 4(a). It is immediately apparent from this map that for each

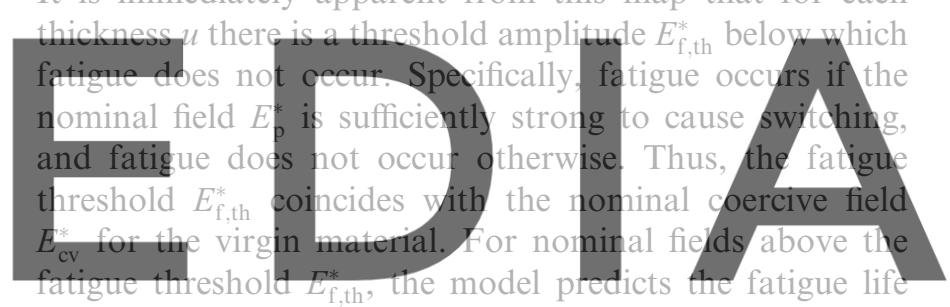
$N_{\mathrm{f}}$ to shorten with increasing nominal field amplitude $E_{\mathrm{p}}^{*}$.

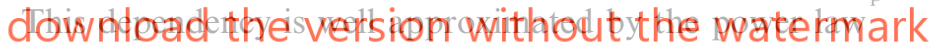
$N_{\mathrm{f}}=A E_{\mathrm{p}}^{* n}$

with exponent $-n \approx 2.8$.

For bulk-like samples and high frequencies, the fatigue life has a steeper dependency on the applied field, as shown in Fig. 5(a). It is readily verified that the transition from non-switching to switching bulk behavior, as the applied field increases, appears smoother as the field frequency increases, thus leading to the calculated fatigue behavior. In addition, $E_{\mathrm{f}, \mathrm{th}}^{*}$ can be greatly in excess of $E_{\mathrm{cv}}^{*}$, as Fig. 5(b) shows. It also indicates that the low frequency limit is attained for the lowest frequency used.

Corresponding experimental data for smooth tetragonal PZT samples $[39,1,40]$ are shown in Fig. 4(b) for purposes of comparison. Evidently, the predicted existence of a fatigue threshold and the reduction in fatigue life with nominal field amplitude are consistent with the data. The nominal fatigue threshold $E_{\mathrm{f}, \mathrm{th}}^{*}$ is indeed observed to be greater than or equal to the nominal coercive field $E_{\mathrm{cv}}^{*}$, as obtained from the respective hysteresis loops. Besides these experiments where no microcracking was observed or analyzed, the relation between switching and fatigue has also been established on indented and precracked samples by the experiments of $\mathrm{Zhu}$ et al. [22]. Thus, switching appears as a necessary (though not suffi- 


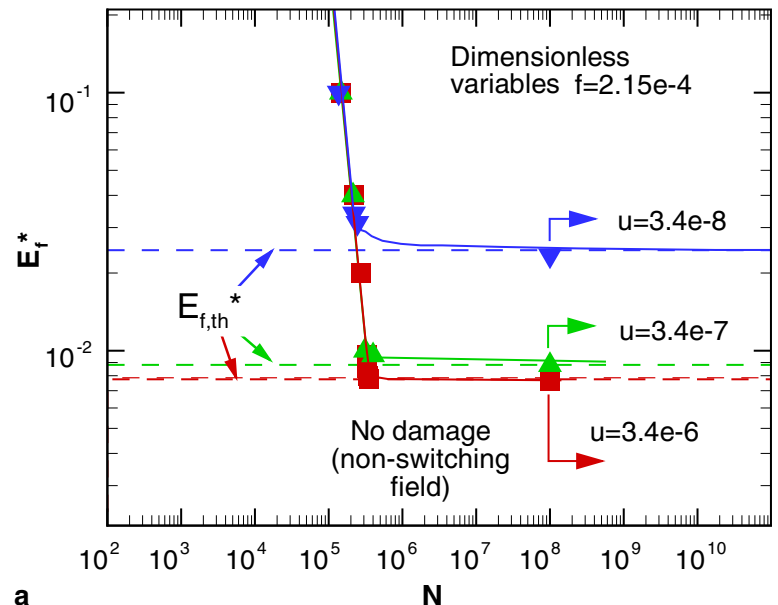

a

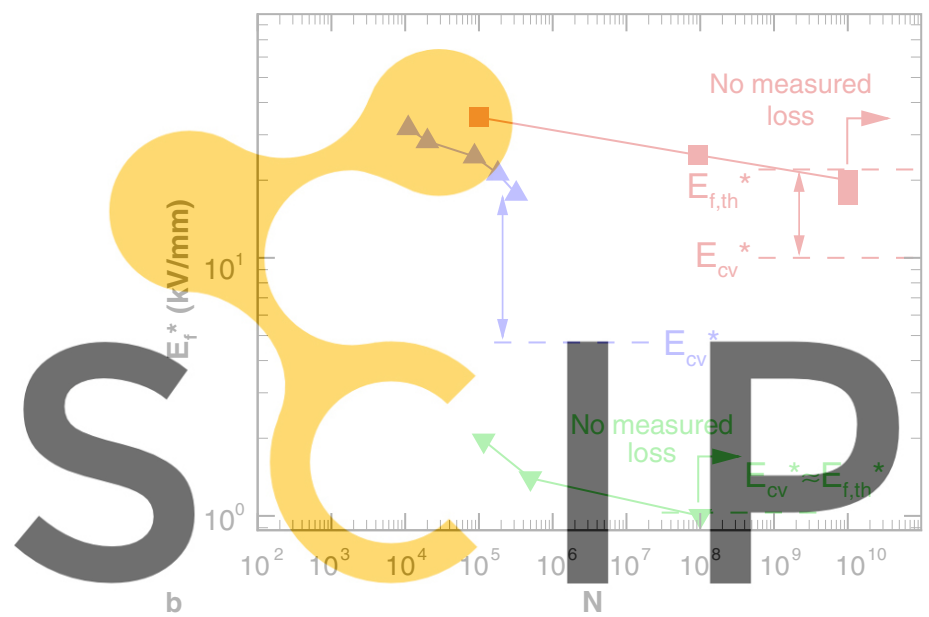

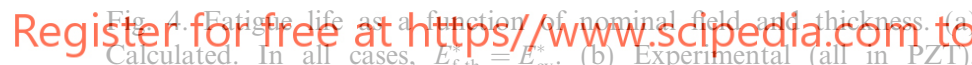
loss $=10 \%$. $\mathbf{a}$ : Grossmann et al. [39], V: Nuffer et al. [1], $\mathbf{\Delta}$ : Mihara et al. [40]. In all cases, $E_{\mathrm{r}, \mathrm{th}}^{*} \geqslant E_{\mathrm{cv}}^{*}$

cient) condition for electrical fatigue, regardless of the fatigue mechanism operating. It should be noted that there are other methods, alternative to electric fatigue, to suppress polarization (e.g., [41]). The experimental data shown in Fig. 4(b) is also suggestive of power-law behavior (see Eq. (35)) with exponents in the range $-n \approx 0.16$ 0.24 . The difference with the predicted value is due to the choice bulk behavior, which has no dependence of the actual coercive field on actual field amplitude for $E_{\mathrm{p}}>E_{\mathrm{c}}$, and not to the cohesive model. The dependence of the fatigue threshold on the applied-field frequency, with high frequencies delaying the onset of fatigue, was established by Grossmann et al. [39].

It is worth mentioning that some alternatives were developed to suppress polarization fatigue, including using conducting oxide electrodes [42]. Such devices can be modeled by an interface with a large value of $\delta_{\mathrm{a}}$, which would lead to a minor fatigue effect. In the limit of $\delta_{\mathrm{a}} \rightarrow \infty$, fatigue would be completely absent.

In order to exhibit the size effect predicted by the model, Fig. 6(a) plots specimen thickness against the minimum
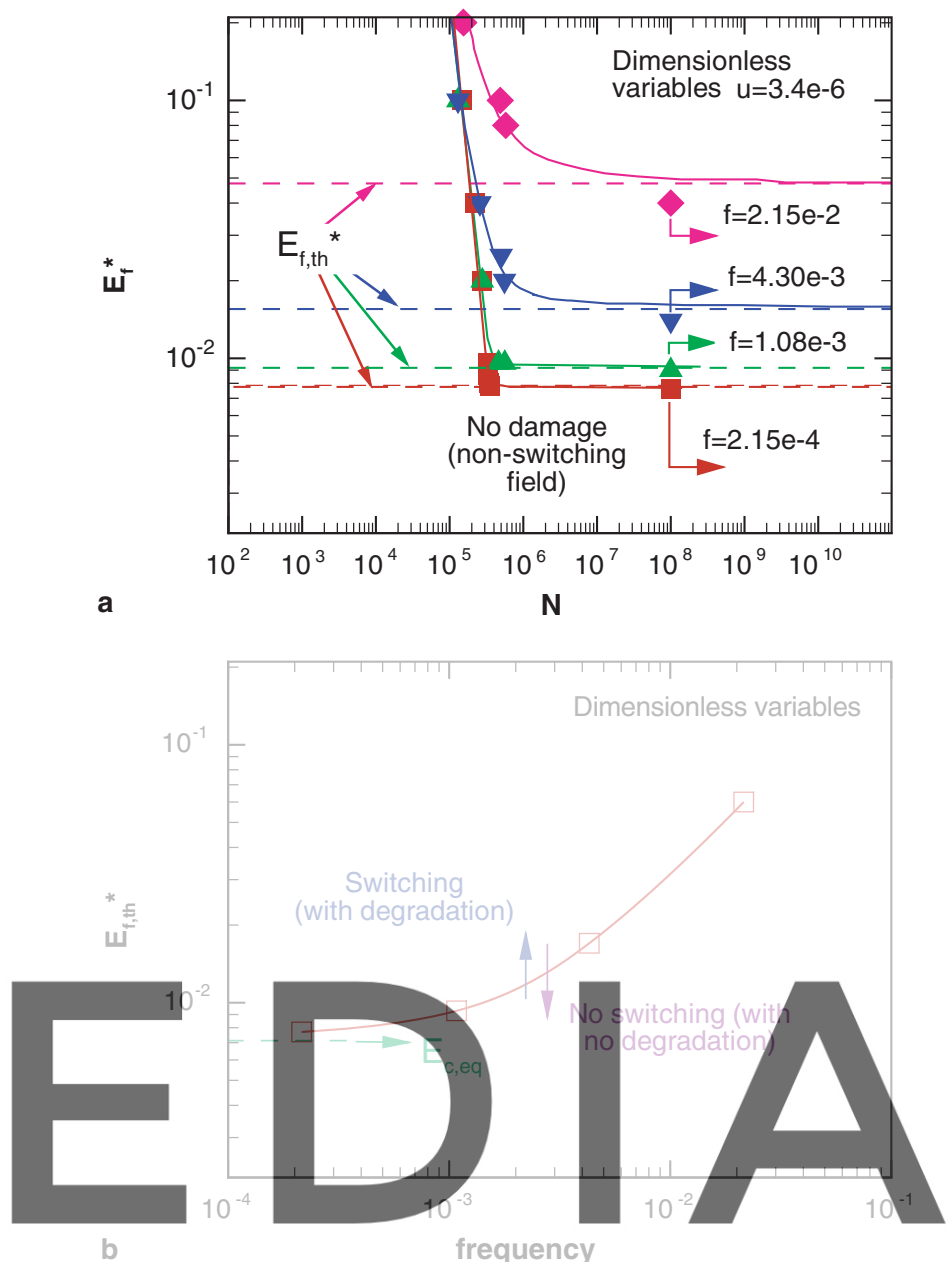

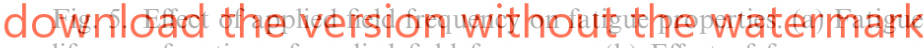
life as a function of applied field frequency. (b) Effect of frequency on nominal threshold field.

value of $E_{\mathrm{p}}^{*}$ for which switching occurs, and the maximum value of $E_{\mathrm{p}}^{*}$ for which switching does not occur. Insight into this dependence may be derived by analyzing the equilibrium case of $\dot{P}=0$ in Eq. (22). In this case, the governing equations reduce to

$E=a_{1} P+\left(a_{2}-\frac{b_{1}^{2}}{2 c_{1}}\right) P^{3}+a_{3} P^{5}$,

$P=E\left(K_{\mathrm{u}} \gamma^{2} u-\epsilon_{0}\right)+K_{\mathrm{u}} \gamma^{2} \Delta \phi_{T}$.

For a virgin material, i.e., a material with high $K_{\mathrm{u}}$, it is readily shown that the nominal coercive field $E_{\mathrm{cv}}^{*}$ required for switching is of the form

$E_{\mathrm{cv}}^{*}(u)=\Delta \phi_{\mathrm{c}}(u) / u \sim E_{\text {ceq }}+\left(P_{\text {ceq }}+\epsilon_{0} E_{\text {ceq }}\right) /\left(K_{\mathrm{u}} \gamma^{2} u\right)$.

where $E_{\text {ceq }}=0.007129$. This relation is plotted in Fig. 6(a) along with the exact calculated values of $E_{\mathrm{cv}}^{*}(u)$ from Eq. (36a). It is noteworthy that $E_{\mathrm{cv}}^{*}$ decreases monotonically with size $u$ to a limiting value $E_{\text {ceq }}$ characteristic of large components. Conversely, $E_{\mathrm{cv}}^{*}$ increases as $u^{-1}$ for a small component thickness. This prediction of the model is born out by the experimental data, Fig. 6, which is 

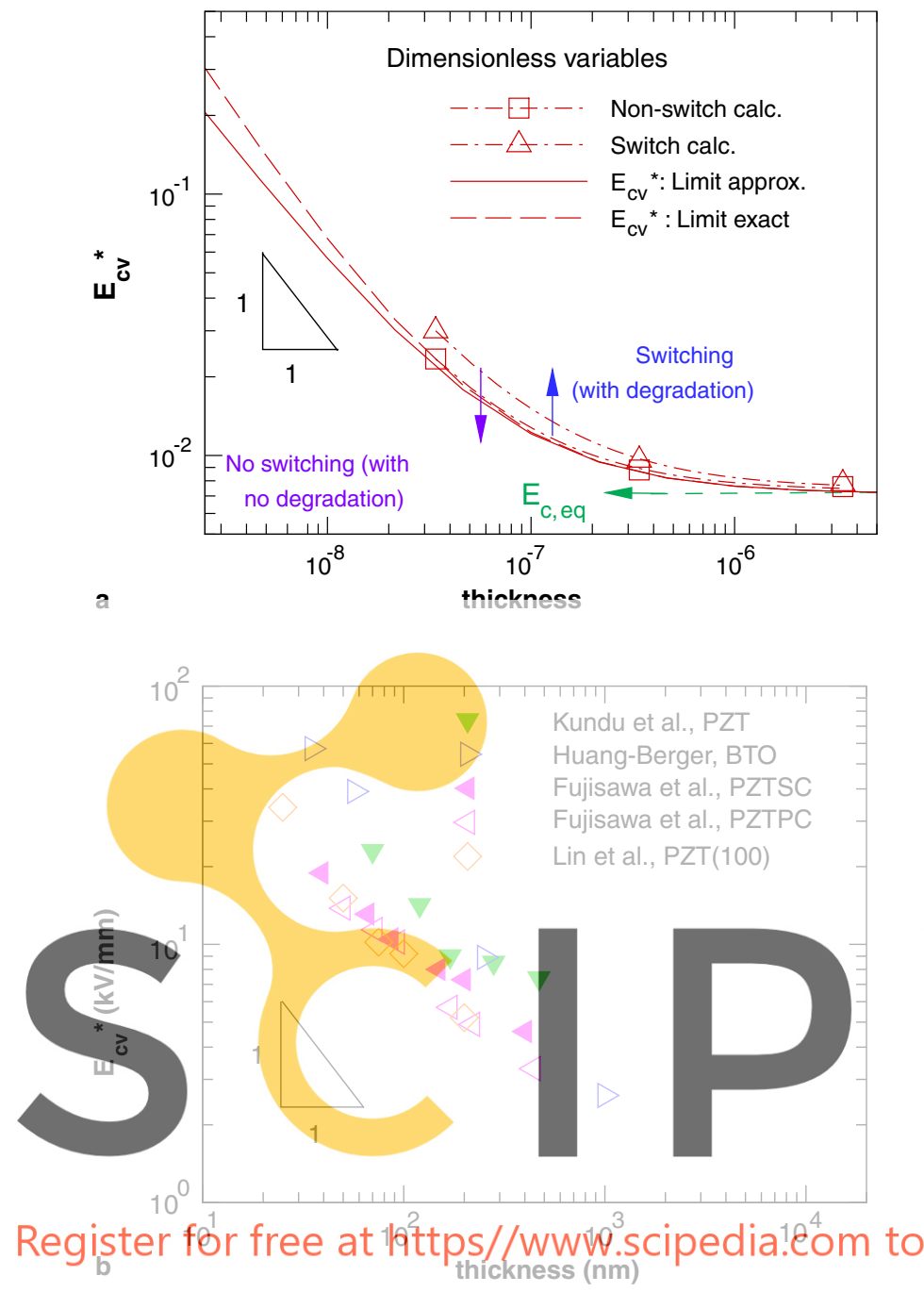

Fig. 6. Nominal electric field required for switching. (a) Calculated. Determined from Fig. 4(a): $\triangle$ : lowest values of $E_{\mathrm{p}}^{*}$ with switching behavior, $\square$ : highest values of $E_{\mathrm{p}}^{*}$ with non-switching behavior. Determined from models with $K_{1}=K_{\mathrm{u}}=$ const.: - : approximate model, -- :

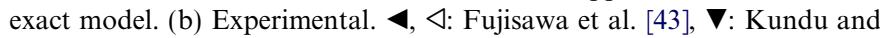
Lee [44], $\triangleright$ : Huang and Berger [45], $\diamond$ : Lin et al. [46].

consistent with the $E_{\mathrm{cv}}^{*} \sim u^{-1}$ scaling behavior. By contrast, the size effect above the nominal fatigue threshold $E_{\mathrm{f}, \mathrm{th}}^{*}$ is negligible, Fig. 4(a). This lack of sensitivity to the component size is also visible in the evolution of $\delta_{\mathrm{p}}$, Fig. 3. Thus, the main effect of component size is on the ability of the material to switch and, by extension, on the nominal fatigue threshold $E_{\mathrm{f}, \mathrm{th}}^{*}$. It should be noted that alternative explanations for the size effect have been proposed, including depolarization fields [47,48], epitaxial stress effects $[49,45]$ and variants of a conductive layer next to the ferroelectric-electrode interface $[50,51]$.

\section{Summary and concluding remarks}

We have presented a model of electro-mechanical ferroelectric fatigue based on the postulate of a ferroelectric cohesive law that: couples mechanical displacement and electric-potential discontinuity to mechanical tractions and surface-charge density; and exhibits a monotonic envelope and loading-unloading hysteresis. In conjunction with a constitutive model accounting for domain switching, the electro-mechanical cohesive fatigue law is able to induce ferroelectric fatigue by the following mechanism: as degradation proceeds, the surface of electromechanical discontinuity absorbs an increasingly large amount of the displacement and/or electric potential difference, thereby unloading the bulk and hindering switching. We identify the end of the fatigue life with the time at which the material loses its ability to sustain loading/ applied field cycles of a certain constant amplitude. We have compared selected predictions of the model with experimental data for a simple test configuration consisting of an infinite slab acted upon by an oscillatory voltage differential across the slab and otherwise stress free. The model captures salient features of the experimental record including: the existence of a threshold nominal field for the onset of fatigue; the dependence of the threshold on the applied-field frequency; the dependence of fatigue life on the amplitude of the nominal field; and the dependence of the coercive field, and thus of the fatigue threshold, on the size of the component, or
size effect. Our results seen to indicate that planar-like
regions affected by cycling may lead to the obscrved fati-
gue in tetragonal PZT.
The ability of the model to predict the goserved size
effect stems directly from the fact that cohesive laws intro-
duce a characteristic or intrinsic material length scale. Hence, in the present model the size effect is a material

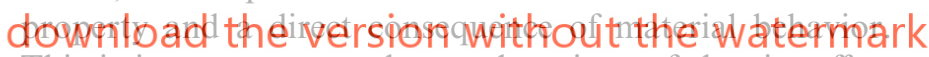
This is in contrast to other explanations of the size effect found in the literature (e.g., $[50,51,49])$, where the length scale is often structural.

In closing, we emphasize that the primary focus of this study has been to investigate qualitative trends and no systematic attempt has been made to optimize fit to the experimental data. It is conceivable that good quantitative agreement with the data could be obtained by some extensions and careful calibration of the model, including full finite element calculations; the use of more accurate bulk constitutive relations; and a systematic identification of aspects of the cohesive law such as the precise shape of the monotonic envelope and of the loading-unloading law.

\section{Acknowledgment}

The financial support of the Army Research Office under MURI Grant No. DAAD19-01-1-0517 is gratefully acknowledged.

\section{References}

[1] Nuffer J, Lupascu DC, Rödel J. Damage evolution in ferroelectric PZT induced by bipolar electric cycling. Acta Mater 2000;48: 3783-94. 
[2] Tagantsev AK, Stolichnov I, Colla EL, Setter N. Polarization fatigue in ferroelectric films: basic experimental findings, phenomenological scenarios, and microscopic features. J Appl Phys 2001;90(3): $1387-402$.

[3] Robels U, Arlt G. Domain wall clamping in ferroelectrics by orientation of defects. J Appl Phys 1993;73(7):3454-60.

[4] Warren WL, Dimos D, Tuttle BA, Nasby RD, Pike GE. Electronic domain pinning in $\mathrm{Pb}(\mathrm{Zr}, \mathrm{Ti}) \mathrm{O}_{3}$ thin films and its role in fatigue. Appl Phys Lett 1994;65(8):1018-20.

[5] Scott JF, Araujo CA, Melnick BM, Mcmillan LD, Zuleeg R. Quantitative measurement of space-charge effects in lead zirconatetitanate memories. J Appl Phys 1991;70(1):382-8.

[6] Kim SJ, Jiang Q. Microcracking and electric fatigue of polycrystalline ferroelectric ceramics. Smart Mater Struct 1996;5(3):321-6.

[7] Stolichnov I, Tagantsev A, Setter N, Cross JS, Tsukada M. Topinterface-controlled switching and fatigue endurance of $(\mathrm{Pb}, \mathrm{La})$ (Zr,Ti)O ${ }_{3}$ ferroelectric capacitors. Appl Phys Lett 1999;74(23):3552-4.

[8] Warren WL, Dimos D, Waser RM. Degradation mechanisms in ferroelectric and high-permittivity perovskites. MRS Bull 1996;21(7): $40-5$

[9] Damjanovic D. Ferroelectric, dielectric and piezoelectric properties of ferroelectric thin films and ceramics. Rep Prog Phys 1998;61(9) 1267-324

10] Colla EL, Taylor DV, Tagantsev AK, Setter N. Discrimination between bulk and interface scenarios for the suppression of the switchable polarization (fatigue) in $\mathrm{Pb}(\mathrm{Zr}, \mathrm{Ti}) \mathrm{O}_{3}$ thin films capacitors with Pt electrodes. Appl Phys Lett 1998;72(19):2478-80

[11] Dawber M. Scott JF. A model for fatigue in ferroelectric perovskite

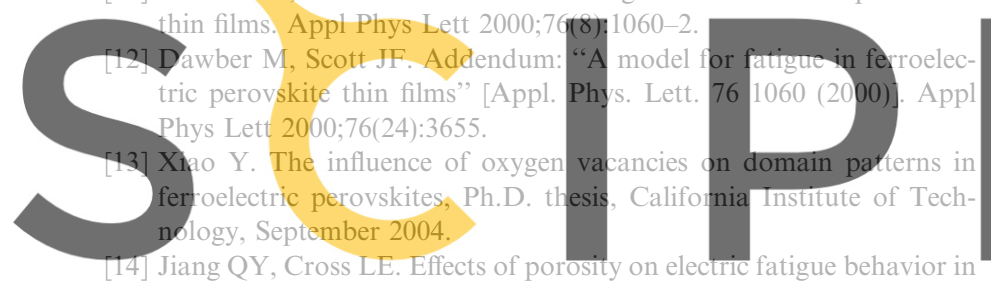
PLZT and PZT ferroelectric ceramics. J Mater Sci 1993;28(16):

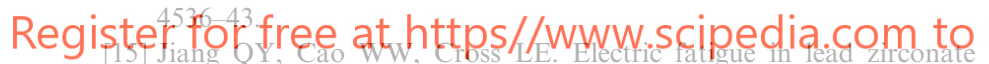
titanate ceramics. J Am Ceram Soc 1994;77(1):211-5

[16] Nuffer J, Lupascu DC, Rodel J. Microcrack clouds in fatigued electrostrictive 9.5/65/35 PLZT. J Eur Ceram Soc 2001;21(10-11): 1421-3.

[17] Nuffer J, Lupascu DC, Glazounov A, Kleebe H, Rödel J. Microstructural modifications of ferroelectric lead zirconate titanate ceramics due to bipolar electric fatigue. J Eur Ceram Soc 2002;22(13):2133-42.

[18] Burcsu E. Investigation of large strain actuation in barium titanate, $\mathrm{Ph} . \mathrm{D}$. thesis, California Institute of Technology (May 2001).

[19] Cao HC, Evans AG. Electric-field-induced fatigue crack growth in piezoelectrics. J Am Ceram Soc 1994;77(7):1783-6.

[20] Lynch CS, Chen L, Suo Z, McMeeking RM, Yang W. Crack growth in ferroelectric ceramics driven by cyclic polarization switching. $\mathbf{J}$ Intell Mater Syst Struct 1995;6(2):191-8.

[21] Lynch CS, Yang W, Collier L, Suo Z, McMeeking RM. Electric field induced cracking in ferroelectric ceramics. Ferroelectrics 1995;166: $11-30$.

[22] Zhu T, Fang F, Yang W. Fatigue crack growth in ferroelectric ceramics below the coercive field. J Mater Sci Lett 1999;18: 1025-7.

[23] Fang D, Ai KS, Liu J. Electromechanical deformation and fracture of piezoelectric/ferroelectric materials. Acta Mech Sin 2001;17:193213.

[24] Shaw TM, Trolier-McKinstry S, McIntyre PC. The properties of ferroelectric films at small dimensions. Ann Rev Mater Sci 2000;30:263-98.

[25] Nguyen O, Repetto EA, Ortiz M, Radovitzky R. A cohesive model of fatigue crack growth. Int J Fract 2001;110(4):351-69.
[26] Serebrinsky S, Ortiz M. A hysteretic cohesive-law model of fatiguecrack nucleation. Scripta Mater 2005;53(10):1193-6.

[27] Gao HJ, Zhang TY, Tong P. Local and global energy release rates for an electrically yielded crack in a piezoelectric ceramic. J Mech Phys Sol 1997;45(4):491-510.

[28] Fulton CC, Gao H. Microstructural modeling of ferroelectric fracture. Acta Mater 2001;49(11):2039-54.

[29] Fulton CC, Gao HJ. Effect of local polarization switching on piezoelectric fracture. J Mech Phys Sol 2001;49(4):927-52.

[30] Ortiz M, Pandolfi A. Finite deformation irreversible cohesive elements for three-dimensional crack-propagation analysis. Int $\mathbf{J}$ Numer Meth Eng 1999;44(9):1267-82.

[31] Suo Z, Kuo C-M, Barnett DM, Willis JR. Fracture mechanics for piezoelectric ceramics. J Mech Phys Sol 1992;40(4):739-65.

[32] Landis CM. A new finite-element formulation for electromechanical boundary value problems. Int J Numer Meth Eng 2002;55(5): 613-28.

[33] Suo Z, Gong X. Reliability of ceramic multilayer actuators: a nonlinear finite element simulation. J Mech Phys Sol 1996:44: $751-69$.

[34] Shu YC, Bhattacharya K. Domain patterns and macroscopic behaviour of ferroelectric materials. Philos Mag B 2001;81(12): 2021-54

[35] Li S. On saturation-strip model of a permeable crack in a piezoelectric ceramic. Acta Mech 2003;165(1-2):47-71.

[36] McMeeking RM. Towards a fracture mechanics for brittle piezoelectric and dielectric materials. Int J Fract 2001;108(1):2541

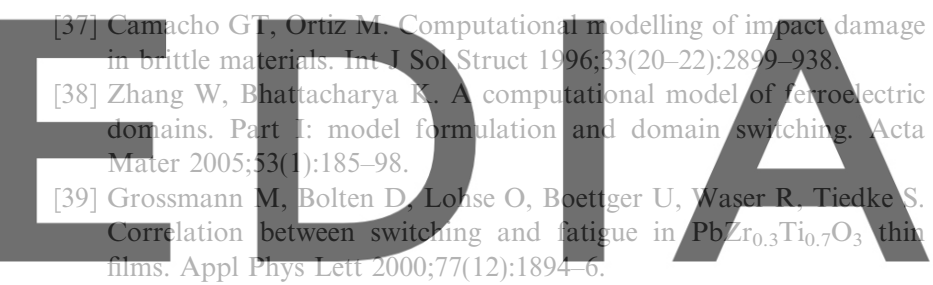

401 Mihara T. Watanabe H. De Araujo CAP. Polarization fatigue

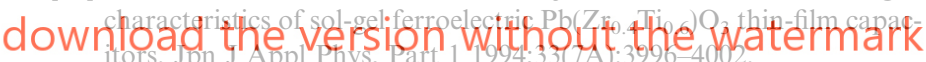

[41] Warren WL, Dimos D, Tuttle BA, Pike GE, Schwartz RW, Clews PJ, Mcintyre DC. Polarization suppression in $\mathrm{Pb}(\mathrm{Zr}, \mathrm{Ti}) \mathrm{O}_{3}$ thin-films. J Appl Phys 1995;77(12):6695-702.

[42] Lee JJ, Thio CL, Desu SB. Electrode contacts on ferroelectric $\mathrm{Pb}\left(\mathrm{Zr}_{x} \mathrm{Ti}_{1-x}\right) \mathrm{O}_{3}$ and $\mathrm{SrBi}_{2} \mathrm{Ta}_{2} \mathrm{O}_{9}$ thin films and their influence on fatigue properties. J Appl Phys 1995;78(8):5073-8.

[43] Fujisawa H, Nakashima S, Kaibara K, Shimizu M, Niu H. Size effects of epitaxial and polycrystalline $\mathrm{Pb}(\mathrm{Zr}, \mathrm{Ti}) \mathrm{O}_{3}$ thin films grown by metalorganic chemical vapor deposition. Jpn J Appl Phys, Part 1 1999;38(9B):5392-6.

[44] Kundu TK, Lee JY-M. Thickness dependence of the time dependent dielectric breakdown characteristics of $\mathrm{Pb}(\mathrm{Zr}, \mathrm{Ti}) \mathrm{O}_{3}$ thin film capacitors for memory device applications. Jpn J Appl Phys, Part 1 2000;39(6A): 3488-91.

[45] Huang GF, Berger S. Combined effect of thickness and stress on ferroelectric behavior of thin $\mathrm{BaTiO}_{3}$ films. J Appl Phys 2003;93(5): 2855-60.

[46] Lin CH, Friddle PA, Ma CH, Daga A, Chen HD. Effects of thickness on the electrical properties of metalorganic chemical vapor deposited $\mathrm{Pb}(\mathrm{Zr}, \mathrm{Ti}) \mathrm{O}_{3}(25-100 \mathrm{~nm})$ thin films on $\mathrm{LaNiO}_{3}$ buffered Si. J Appl Phys 2001;90(3):1509-15.

[47] Wurfel P, Batra IP. Depolarization-field-induced instability in thin ferroelectric films - Experiment and theory. Phys Rev B 1973;8(11): 5126-33.

[48] Mehta RR, Silverman BD, Jacobs JT. Depolarization fields in thin ferroelectric films. J Appl Phys 1973;44(8):3379-85.

[49] Pertsev NA, Rodríguez Contreras J, Kukhar VG, Hermanns B, Kohlstedt $\mathrm{H}$, Waser R. Coercive field of ultrathin $\mathrm{PbZr}_{0.52} \mathrm{Ti}_{0.48} \mathrm{O}_{3}$ epitaxial films. Appl Phys Lett 2003;83(16):3356-8. 
[50] Cillessen JFM, Prins MWJ, Wolf RM. Thickness dependence of the switching voltage in all-oxide ferroelectric thin-film capacitors prepared by pulsed laser deposition. J Appl Phys 1997;81(6): 2777-83.
[51] Tagantsev AK, Stolichnov IA. Injection-controlled size effect on switching of ferroelectric thin films. Appl Phys Lett 1999;74(9): $1326-8$.
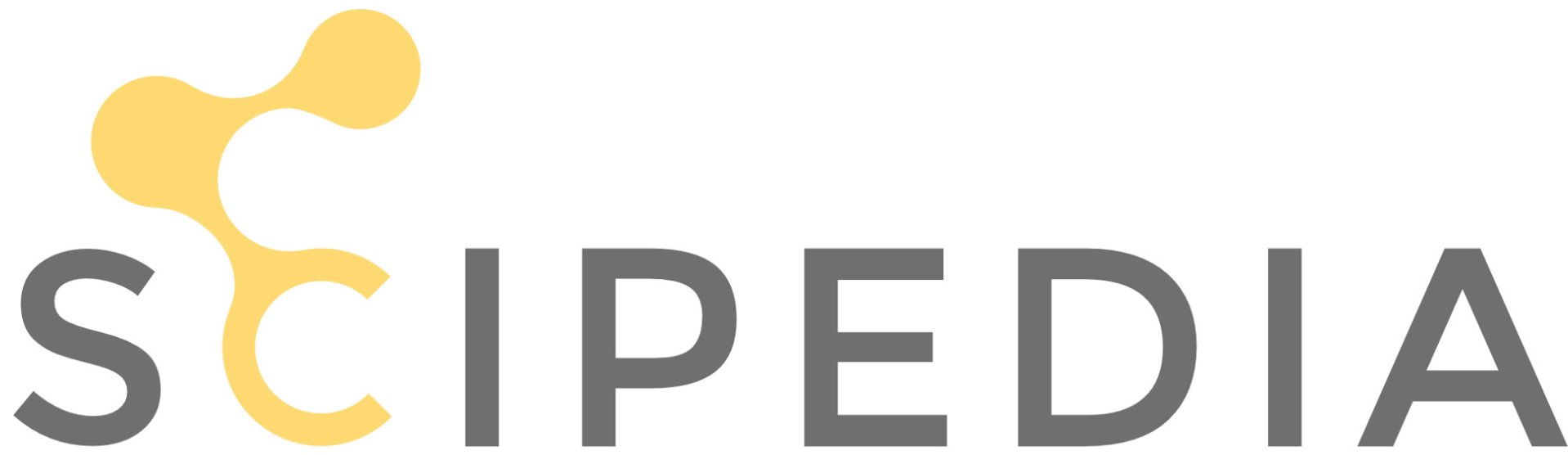

Register for free at https//www.scipedia.com to download the version without the watermark 